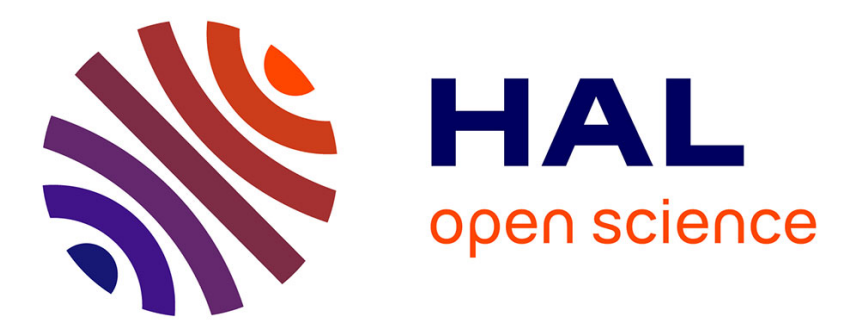

\title{
A poroelastic model valid in large strains with applications to perfusion in cardiac modeling
}

Dominique Chapelle, Jacques Sainte-Marie, Jean-Frédéric Gerbeau, Irene

Vignon-Clementel

\section{To cite this version:}

Dominique Chapelle, Jacques Sainte-Marie, Jean-Frédéric Gerbeau, Irene Vignon-Clementel. A poroelastic model valid in large strains with applications to perfusion in cardiac modeling. Computational Mechanics, 2010, 46 (1), pp.91-101. 10.1007/s00466-009-0452-x . inria-00542672

\section{HAL Id: inria-00542672 \\ https://hal.inria.fr/inria-00542672}

Submitted on 22 Jun 2013

HAL is a multi-disciplinary open access archive for the deposit and dissemination of scientific research documents, whether they are published or not. The documents may come from teaching and research institutions in France or abroad, or from public or private research centers.
L'archive ouverte pluridisciplinaire HAL, est destinée au dépôt et à la diffusion de documents scientifiques de niveau recherche, publiés ou non, émanant des établissements d'enseignement et de recherche français ou étrangers, des laboratoires publics ou privés. 


\title{
A poroelastic model valid in large strains with applications to perfusion in cardiac modeling
}

\author{
D. Chapelle ${ }^{1}$, J.-F. Gerbeau ${ }^{1}$, J. Sainte-Marie ${ }^{1,2}$ and I. Vignon-Clementel ${ }^{1}$ \\ 1 INRIA Paris-Rocquencourt, BP 105, 78153 Le Chesnay Cedex, France \\ 2 Saint-Venant Laboratory, 6 quai Watier, 78401 Chatou Cedex, France
}

In Press

\begin{abstract}
This paper is motivated by the modeling of blood flows through the beating myocardium, namely cardiac perfusion. As in other works, perfusion is modeled here as a flow through a poroelastic medium. The main contribution of this study is the derivation of a general poroelastic model valid for a nearly incompressible medium which experiences finite deformations. A numerical procedure is proposed to iteratively solve the porous flow and the nonlinear poroviscoelastic problems. Three-dimensional numerical experiments are presented to illustrate the model. The first test cases consist of typical poroelastic configurations: swelling and complete drainage. Finally, a simulation of cardiac perfusion is presented in an idealized left ventricle embedded with active fibers. Results show the complex temporal and spatial interactions of the muscle and blood, reproducing several key phenomena observed in cardiac perfusion.
\end{abstract}

\section{Introduction}

Despite recent advances on the anatomical description and measurements of the coronary tree - see e.g. $[1,2]$ - and on the corresponding physiological, physical and numerical modeling aspects - see e.g. [3-5] - the complete modeling and simulation of blood flows inside the coronaries from the arteries to the veins via the capillaries is still out of reach. Therefore, in order to model blood perfusion in the cardiac tissue, we must limit the description of the detailed flows at a given space scale, and simplify the modeling of the smaller scale flows by aggregating these phenomena into macroscopic quantities, by some kind of "homogenization" procedure. To that purpose, the modeling of the fluid-solid coupling within the framework of porous media appears appropriate.

Poromechanics is a simplified mixture theory where a complex fluid-structure interaction problem is replaced

Send offprint requests to: I. Vignon-Clementel by a superposition of both components, each of them representing a fraction of the complete material at every point. It originally emerged in soils mechanics with the work of Terzaghi [6], and Biot [7,8] later gave a description of the mechanical behavior of a porous medium using an elastic formulation for the solid matrix, and Darcy's law for the fluid flow through the matrix. Finite strain poroelastic models have already been proposed [912 , albeit with ad hoc formulations for which compatibility with thermodynamics laws and incompressibility conditions is not established. Poroelastic models have also been considered in the framework of fluid-structure interaction, e.g. to model blood vessel walls [13], with some extensions including lipid (LDL) [14] and drug [15, 16] transport. Other formulations have been proposed with multiple fluid compartments - or a continuum of such compartments - coupled with each other and with the solid medium [17-19], and with applications to the modeling of living tissues [20], but of course such models are extremely difficult to validate with experimental evidence in practice.

In this article, we introduce a general poroelastic formulation valid for finite strains and compatible with incompressibility, as these two features are deemed to be important in the modeling of living tissues. We follow the strategy - presented in [21] in a linear framework - of deriving the formulation from an appropriate free energy functional, which is crucial to guarantee that fundamental thermodynamics principles are satisfied. We then propose a numerical procedure to solve the resulting system of equations and we illustrate the behavior of this poroelastic model by several numerical examples. In the fourth section, we particularize the illustration to cardiac perfusion phenomena.

\section{Porous mechanics}

In this section, we first introduce kinematical and conservation laws of the porous medium. Based on thermodynamics considerations we then develop the constitutive 
laws that characterize the solid phase, the fluid phase and their interaction.

\subsection{Kinematical, conservation and balance laws}

The saturated porous continuum consists of a solid part - referred to as the "skeleton" - and a fluid part that accounts for fractions $\phi_{0}$ and $\phi$ of the combined volume in the reference and deformed configurations, respectively. Throughout this paper, the subscript " 0 " will be used to refer to the reference configuration. Let $y$ be the displacement field of the skeleton as defined in a Lagrangian formulation, namely, given with respect to a fixed reference configuration. Then

$$
\underline{\underline{F}}=\underline{\underline{1}}+\underline{\underline{\nabla}}_{\hat{x}} \underline{y}
$$

is the deformation gradient and

$$
\underline{\underline{C}}=\underline{\underline{F}}^{T} \cdot \underline{\underline{F}}, \quad \underline{\underline{e}}=\frac{1}{2}(\underline{\underline{C}}-\underline{\underline{1}})
$$

are respectively the right Cauchy-Green deformation tensor and the Green-Lagrange strain tensor. Note that we have used $\hat{x}$ as a subscript in the definition of $\underline{F}$ to emphasize that we are taking the gradient with respect to the reference configuration, namely, the Lagrangian gradient. We recall that the three classical strain invariants are then given by

$$
I_{1}=\operatorname{tr} \underline{\underline{C}}, \quad I_{2}=\frac{1}{2}\left((\operatorname{tr} \underline{\underline{C}})^{2}-\operatorname{tr} \underline{\underline{C^{2}}}\right), \quad I_{3}=\operatorname{det} \underline{\underline{C}},
$$

while the reduced invariants are defined by

$$
J_{1}=I_{1} I_{3}^{-\frac{1}{3}}, \quad J_{2}=I_{2} I_{3}^{-\frac{2}{3}}, \quad J=I_{3}^{\frac{1}{2}}=\operatorname{det} \underline{\underline{F}} .
$$

Denoting indifferently by a dot symbol or by $\frac{d}{d t}$ the Lagrangian time derivatives - namely, obtained when following "solid particles" - geometrical considerations lead to

$$
J \nabla \cdot \underline{u}^{s}=\frac{d J}{d t}
$$

where

$$
\underline{u}^{s}=\underline{\dot{y}}
$$

is the velocity of a solid particle. Note that the divergence is here considered in Eulerian form. Denoting by $m$ the increase in fluid mass per unit volume of the reference configuration, a straightforward conservation argument gives

$$
m=\rho^{f} J \phi-\rho_{0}^{f} \phi_{0},
$$

where $\rho^{f}$ is the fluid mass per unit of fluid volume. Furthermore, conservation of fluid mass is in Eulerian form:

$$
\frac{\partial}{\partial t}\left(\rho^{f} \phi\right)+\nabla \cdot\left(\rho^{f} \phi \underline{u}^{f}\right)=\rho^{f} s
$$

with $\underline{u}^{f}$ the velocity of a fluid particle, and $s$ a general sink or source term. Combining the equations (1) and (4) with the definition of the perfusion velocity

$$
\underline{w}=\phi\left(\underline{u}^{f}-\underline{u}^{s}\right),
$$

and of the added mass $m$ in (3), we obtain the general conservation of fluid mass in porous media

$$
\nabla \cdot\left(\rho^{f} \underline{w}\right)+\frac{1}{J} \frac{d m}{d t}=\rho^{f} s .
$$

In addition, conservation of mass of the solid phase can be written as

$$
J \rho^{s}(1-\phi)=\rho_{0}^{s}\left(1-\phi_{0}\right) .
$$

We conclude this section with the balance of momentum of the porous medium written in Lagrangian form:

$$
\left(\rho_{0}+m\right) \underline{\ddot{y}}=\nabla_{\hat{x}} \cdot(\underline{\underline{F}} \cdot \underline{\underline{\Sigma}}) .
$$

In the inertia term, we have neglected the discrepancy between the fluid and solid accelerations, and $\rho_{0}$ denotes the total mass per unit volume in the reference configuration, i.e.

$$
\rho_{0}=\phi_{0} \rho_{0}^{f}+\left(1-\phi_{0}\right) \rho_{0}^{s}
$$

\subsection{Constitutive laws}

In this section we propose some constitutive equations to adequately represent poromechanics in finite strain in a formulation compatible with the incompressible limit.

Thermodynamics considerations $[21,22]$ entail the existence of a free energy $\Psi=\Psi(\underline{e}, m, T)$, where $T$ denotes the temperature. In the absence of internal dissipation we then have

$$
\underline{\underline{\Sigma}}=\frac{\partial \Psi}{\partial \underline{\underline{e}}}, \quad g_{m}=\frac{\partial \Psi}{\partial m}
$$

where $\Sigma$ denotes the second Piola-Kirchhoff stress tensor and $g_{m}(p, T)$ the free enthalpy of the unit fluid mass, which characterizes the fluid constitutive behavior through

$$
\frac{1}{\rho^{f}}=\frac{\partial g_{m}}{\partial p},
$$

where $p$ is the pressure in the fluid - also called "interstitial pressure". The constitutive behavior of the poroelastic material is then entirely characterized by the choice of the functionals $\Psi$ and $g_{m}$. Assuming an isothermal regime, we propose to extend the linear theory of [21] by considering

$$
\begin{gathered}
\Psi=W^{h y p}(\underline{\underline{e}})-M b \frac{m}{\rho_{0}^{f}}(J-1) f(J) \\
+\frac{1}{2} M\left(\frac{m}{\rho_{0}^{f}}\right)^{2} f(J), \\
g_{m}=\frac{p-p_{0}}{\rho_{0}^{f}} .
\end{gathered}
$$


In the expression (10), $W^{\text {hyp }}$ denotes some hyperelastic potential to be chosen, $M$ is the so-called Biot modulus and $b$ a parameter characteristic of the skeleton. Note that the choice (11) directly implies with (9) that

$$
\rho^{f}=\rho_{0}^{f},
$$

namely, the fluid considered is incompressible. Concerning (10) the most straightforward extension of the linear theory would correspond to $f(J)=1$, but we will see that this choice is in general incompatible with incompressible conditions, by which we now mean incompressibility of the fluid and the solid individually.

With the choices (10)-(11) the direct application of (8)-(9) then gives

$$
\begin{gathered}
\underline{\underline{\Sigma}}=\frac{\partial W^{h y p}}{\partial \underline{\underline{e}}}-M b \frac{m}{\rho_{0}^{f}}\left(f+(J-1) f^{\prime}\right) J \underline{\underline{C}}^{-1} \\
+\frac{1}{2} M\left(\frac{m}{\rho_{0}^{f}}\right)^{2} f^{\prime} J \underline{\underline{C}}^{-1}, \\
p-p_{0}=M f(J)\left(b(1-J)+\frac{m}{\rho_{0}^{f}}\right) .
\end{gathered}
$$

Using (14) we can substitute $m / \rho_{0}^{f}$ in (13) to obtain the alternative constitutive relation

$$
\begin{aligned}
\underline{\underline{\Sigma}}= & \frac{\partial W^{h y p}}{\partial \underline{\underline{e}}}-M b^{2}\left(f+\frac{1}{2}(J-1) f^{\prime}\right)(J-1) J \underline{\underline{C}}^{-1} \\
& -b\left(p-p_{0}\right) J \underline{\underline{C}}^{-1}+\frac{1}{2} \frac{\left(p-p_{0}\right)^{2}}{M} \frac{f^{\prime}}{f^{2}} J \underline{\underline{C}}^{-1} .
\end{aligned}
$$

In order to be consistent with incompressibility in this relation, the singularity arising from the hyperelastic stress in the incompressible limit - associated with a "large" bulk modulus - must be compensated by the second term in the right-hand side. Indeed, in the poroelastic material incompressibility does not induce constraints on the strains per se, as added fluid can change the apparent volume without violating the incompressible assumption. Hence, we need to adapt the expression of $f(J)$ according to the specific hyperelastic potential considered. In particular, when using - as we will do in the rest of the paper - the modified Ciarlet-Geymonat expression (see [23] for the original formulation)

$$
\begin{aligned}
W^{h y p}= & \kappa_{1}\left(J_{1}-3\right)+\kappa_{2}\left(J_{2}-3\right) \\
& +K(J-1)-K \ln J,
\end{aligned}
$$

where nearly-incompressible materials are obtained by taking $K$ large compared to $\kappa_{1}$ and $\kappa_{2}$, we obtain

$$
\begin{aligned}
\underline{\underline{\Sigma}}= & \frac{\partial W^{M R}}{\partial \underline{\underline{e}}} \\
& +\left[K-M b^{2} J\left(f+\frac{1}{2}(J-1) f^{\prime}\right)\right](J-1) \underline{\underline{C}}^{-1} \\
& -b\left(p-p_{0}\right) J \underline{\underline{C}}^{-1}+\frac{1}{2} \frac{\left(p-p_{0}\right)^{2}}{M} \frac{f^{\prime}}{f^{2}} J \underline{\underline{C}}^{-1}
\end{aligned}
$$

where we denote by $W^{M R}$ the Mooney-Rivlin type potential

$$
W^{M R}=\kappa_{1}\left(J_{1}-3\right)+\kappa_{2}\left(J_{2}-3\right) .
$$

In the form (17) we can easily see that a necessary and sufficient condition for canceling the singularity is to ensure

$$
J\left(f+\frac{1}{2}(J-1) f^{\prime}\right)=1, \quad \forall J,
$$

and have

$$
\kappa_{s}=K-M b^{2}
$$

remain finite when $K$ grows large. This leads to

$$
f(J)=\frac{2(J-1-\ln J)}{(J-1)^{2}}, \quad f(1)=1,
$$

which is easily seen to be smooth around $J=1$, and (17) gives

$$
\begin{aligned}
\underline{\underline{\Sigma}}= & \frac{\partial W^{M R}}{\partial \underline{\underline{e}}}+\kappa_{s}(J-1) \underline{\underline{C}}^{-1} \\
& -b\left(p-p_{0}\right) J \underline{\underline{C}}^{-1}+\frac{1}{2} \frac{\left(p-p_{0}\right)^{2}}{M} \frac{f^{\prime}}{f^{2}} J \underline{\underline{C}}^{-1} .
\end{aligned}
$$

Note that, assuming that $b$ remains finite, keeping $\kappa_{s}$ finite requires that $M$ grows large when $K$ does, which implies by (14) that we should have $b(1-J)+m / \rho_{0}^{f}=$ 0 in the incompressible limit, since the pressure should remain finite. Hence, it is easily seen that this implies

$$
b=1
$$

in the incompressible limit, in order to be compatible with the conservation relation (3) combined with the preservation of solid volume which entails by (6)

$$
J(1-\phi)=1-\phi_{0} .
$$

It is then interesting to rewrite the incompressible limit of (22), namely,

$$
\underline{\underline{\Sigma}}=\frac{\partial W^{M R}}{\partial \underline{\underline{e}}}+\kappa_{s}(J-1) \underline{\underline{C}}^{-1}-\left(p-p_{0}\right) J \underline{\underline{C}}^{-1},
$$

hence contracting by $\underline{\underline{C}} /(3 J)$ to obtain the volumetric part yields

$$
\frac{\underline{\underline{\underline{E}}}: \underline{\underline{\underline{C}}}}{3 J}=\kappa_{s} \frac{J-1}{J}-\left(p-p_{0}\right),
$$

since the Mooney-Rivlin part has no volumetric contribution, by construction. Then, in a stress-free configuration we have in the linearized small displacements framework

$$
p-p_{0}=\kappa_{s} \operatorname{tr} \underline{\underline{\varepsilon}},
$$

which shows that $\kappa_{s}$ can be interpreted as an elasticity modulus for the skeleton.

Remark: As seen from (22), the constitutive law for the perfectly drained poroelastic material - corresponding to $p=p_{0}-$ reduces to

$$
\underline{\underline{\Sigma}}=\frac{\partial W^{M R}}{\partial \underline{\underline{e}}}+\kappa_{s}(J-1) \underline{\underline{C}}^{-1},
$$


whereas in the undrained situation - namely, $m=0$ - we have by (13)

$$
\underline{\underline{\underline{ }}}=\frac{\partial W^{h y p}}{\partial \underline{\underline{e}}}=\frac{\partial W^{M R}}{\partial \underline{\underline{e}}}+K(J-1) \underline{\underline{C}}^{-1} .
$$

Therefore, $\kappa_{s}$ also plays the role of the apparent bulk modulus of the drained material, which remains finite in the incompressible limit when $K$ tends to infinity, since the drained poroelastic material can freely absorb or expel some fluid.

With the above poroelastic constitutive law, we may encounter some difficulties with the evolution of the porosity $\phi$ which is simply deduced from $m$ and $J$ by (3). This may - indeed - lead to the violation of the constraint $0<\phi<1$ which should always hold. Of course, in linearized poroelasticity these difficulties do not occur because it is assumed that $m / \rho_{0}^{f} \ll \phi_{0}$ and $J \approx 1$. Here we can slightly modify the free energy (10) by considering

$$
\begin{aligned}
\Psi= & W^{h y p}(\underline{\underline{e}})-M b \frac{m}{\rho_{0}^{f}}(J-1) f(J) \\
& +\frac{1}{2} M\left(\frac{m}{\rho_{0}^{f}}\right)^{2} f(J)-\kappa_{0} \ln \left(\frac{m}{\rho_{0}^{f}}+\phi_{0}\right),
\end{aligned}
$$

where the additional term will result in $m / \rho_{0}^{f}+\phi_{0}>0$, hence $\phi>0$ by (3). This leaves the constitutive equation (13) unchanged, while (14) is transformed into

$$
p-p_{0}=M f(J)\left(b(1-J)+\frac{m}{\rho_{0}^{f}}\right)-\frac{\kappa_{0}}{m / \rho_{0}^{f}+\phi_{0}} .
$$

This shows that $p-p_{0}$ can become arbitrarily large (in negative values) when $m / \rho_{0}^{f}$ tends to $-\phi_{0}$ from above, as desired. Note that we could also introduce a similar term to enforce $\phi<1$, but this is not needed when considering nearly-incompressible materials, since in the incompressible limit we have (23), hence $1-\phi>0$ is automatically satisfied. For simplicity, in the remainder of the paper, only equations without this additional term are derived.

Finally, the second principle of thermodynamics - the dissipation associated with the transport of fluid mass [21] - leads to the last equation needed to fully characterize the system, also known as Darcy's law:

$$
\underline{w}=-\underline{\underline{K}} \cdot \nabla p
$$

\subsection{System of equations}

The conservation of mass (5) can be rewritten in terms of pressure using (14). Removing intermediate variables, the system of equations characterizing the porous media dynamics follows from (7), (5), (31) and (14): Find $\underline{y}, \underline{w}$, $p$ and $m$ such that

$$
\left\{\begin{array}{l}
\left(\rho_{0}+m\right) \underline{\ddot{y}}=\nabla_{\hat{x}} \cdot(\underline{\underline{F}} \cdot \underline{\underline{\Sigma}}), \\
\nabla \cdot \underline{w}+\frac{1}{J M f} \frac{d p}{d t}-\frac{f^{\prime}}{M f^{2}}\left(p-p_{0}\right) \nabla \cdot \underline{u}^{s}=-b \nabla \cdot \underline{u}^{s}+s, \\
\underline{w}=-\underline{\underline{K}} \cdot \nabla p, \\
p-p_{0}=M f(J)\left(b(1-J)+\frac{m}{\rho_{0}^{f}}\right) .
\end{array}\right.
$$

$\underline{u}^{s}$ is related to $y$ as defined in (2). This system needs to be complemented by adequate boundary conditions that will be specified later.

\section{Numerical procedure}

In this section, we outline the numerical procedures used to discretize the poroelastic model presented above and to solve the resulting coupled fluid/solid finite-dimensional problems.

\subsection{Poroelastic mechanics}

We use a total Lagrangian description and denote by $\hat{\Omega}$ the reference domain corresponding to the poroelastic medium. The usual principle of virtual work then reads

$$
\begin{array}{r}
\int_{\hat{\Omega}}\left(\rho_{0}+m\right) \underline{\ddot{y}} \cdot \underline{\hat{v}} d \hat{\Omega}+\int_{\hat{\Omega}} \underline{\underline{\Sigma}}:\left(d_{y} \underline{\underline{e}} \cdot \underline{\hat{v}}\right) d \hat{\Omega} \\
\quad+\int_{\hat{\Gamma}} P_{\mathrm{v}} \underline{\hat{v}} \cdot \underline{\underline{F}}^{-1} \cdot \underline{\hat{v}} J d \hat{\Gamma}=0, \quad \forall \underline{\hat{v}} \in \hat{\mathcal{V}},
\end{array}
$$

where $\hat{\mathcal{V}}$ denotes a suitable space of displacement test functions and $d_{y} \underline{\underline{e}} \cdot \underline{\hat{v}}$ the linearized strain variation corresponding to the test function $\underline{\hat{v}}$, viz.

$$
\left(d_{y} \underline{\underline{e}} \cdot \underline{\hat{v}}\right)_{i j}=\frac{1}{2}\left(\hat{v}_{i, j}+\hat{v}_{j, i}+\underline{y}_{, i} \cdot \underline{\hat{v}}_{, j}+\underline{y}_{, j} \underline{\hat{v}}_{, i}\right),
$$

while $P_{\mathrm{v}}$ represents some external pressure applied on a part of the boundary denoted by $\hat{\Gamma}$ which normal is $\underline{\hat{\nu}}$. Note that $P_{\mathrm{v}}$ can also be an unknown quantity provided some adequate additional closure relations are given, such as in lumped models representing the blood in cardiac cavities [24]. Added fluid mass per unit volume $m$ is assumed to be known when solving the equation (33) for the displacement field. In the second term of (33) the stress tensor is assumed to be given by the poroelastic constitutive relation (13).

Regarding spatial discretization, the displacement field is approximated using finite elements with linear shape functions. Time discretization of the above dynamical equation is performed by using the energy preserving Newmark scheme. Namely, for each time step 
$t=n \Delta t$, we consider $(33)$ at time $\left(n+\frac{1}{2}\right) \Delta t$ with the following approximation rules for time derivatives

$$
\begin{gathered}
\underline{\ddot{y}}^{n+\frac{1}{2}}=\left[\underline{\dot{y}}^{n+1}-\underline{y}^{n}\right] / \Delta t, \\
\underline{\dot{y}}^{n+\frac{1}{2}}=\left[\underline{\dot{y}}^{n+1}+\underline{\dot{y}}^{n}\right] / 2=\left[\underline{y}^{n+1}-\underline{y}^{n}\right] / \Delta t,
\end{gathered}
$$

and we use for the stress tensor

$$
\underline{\underline{\Sigma}}^{n+\frac{1}{2}}=\underline{\underline{\Sigma}}\left(\underline{\underline{e}}^{n+\frac{1}{2}}, m^{n+\frac{1}{2}}\right) .
$$

We thus obtain - for each time step - a nonlinear system to be solved using a Newton-type procedure.

\subsection{Fluid flow}

The Darcy and conservation of mass equations in (32) lead to a typical porous flow system, but with non conventional coupling terms due to the compliance of the skeleton. We use an Eulerian description and denote by $\Omega$ the domain in the current configuration. Considering a given displacement of the poroelastic medium - associated with the change of volume $J$ - and a given velocity $\underline{u}^{s}$, the weak form is then

$$
\begin{array}{r}
\int_{\Omega} q\left(\nabla \cdot \underline{w}+\frac{1}{J M f} \frac{d p}{d t}+\left(b-\frac{f^{\prime}}{M f^{2}}\left(p-p_{0}\right)\right) \nabla \cdot \underline{u}^{s}\right) \\
=\int_{\Omega} q s d \Omega, \forall q \in \mathcal{Q}, \\
\int_{\Omega} \underline{\underline{K}}^{-1} \underline{w} \cdot \underline{v} d \Omega-\int_{\Omega} p \nabla \cdot \underline{v} d \Omega=-\int_{\Gamma_{p}} p_{b c} \underline{\nu} \cdot \underline{v} d \Gamma, \\
\forall \underline{v} \in \mathcal{V},
\end{array}
$$

where $\mathcal{Q}$ and $\mathcal{V}$ denote suitable spaces of pressure and velocity test functions, respectively, and $\underline{\nu}$ the normal vector at a given point of the boundary surface $\Gamma . p_{b c}$ represents some boundary pressure applied on the part of the boundary denoted by $\Gamma_{p}$. A flux condition $\underline{w} \cdot \underline{\nu}=w_{b c}$ is applied on the other part of the boundary $\Gamma \backslash \Gamma_{p}$. Regarding the finite element spatial discretization, the pressure is approximated with the elementwise constant finite elements $(P 0)$ and the velocity field with Raviart-Thomas elements of order zero ( $R T 0)$, especially known for their good conservation properties (see [25] e.g.). This mixed fluid system is actually solved with a hybrid finite element approach, where the continuity of the fluxes between elements is enforced through a Lagrange multiplier. Time discretization of the pressure and the displacement (to compute $\underline{u}^{s}$ ) is done using an implicit Euler scheme. Once the pressure and the displacement of the skeleton are known, $m$ can then be computed using the algebraic relation (14) .

\subsection{Coupling procedure}

System (32) is solved through an accelerated fixed point algorithm. More precisely, the discrete counterpart of (3.1) and (3.2) are solved iteratively according to the following procedure:

1. Consider $\underline{y}^{n}, p^{n}, \underline{w}^{n}$ and $m^{n}$ known

2. Initialize $\bar{m}_{0}^{n+1}=m^{n}$

3. Given $m_{k}^{n+\frac{1}{2}}=\left(m_{k}^{n+1}+m^{n}\right) / 2$ solve for $\underline{y}_{k+1}^{n+1}$ such that

$$
\begin{aligned}
& \int_{\hat{\Omega}}\left(\frac{2 \rho_{0}}{\Delta t^{2}}\right)\left(\underline{y}_{k+1}^{n+1}-\underline{y}^{n}-\Delta t \underline{\underline{y}}_{k+1}^{n}\right) \cdot \underline{\hat{v}} d \hat{\Omega} \\
& \quad+\int_{\hat{\Omega}} \underline{\underline{\Sigma}}\left(\underline{\underline{e}}_{k+1}^{n+\frac{1}{2}}, m_{k}^{n+\frac{1}{2}}\right):\left(d_{y} \underline{\underline{e}}_{k+1}^{n+\frac{1}{2}} \cdot \underline{\hat{v}}\right) d \hat{\Omega} \\
& \quad+\int_{\hat{\Gamma}} P_{\mathrm{v}}^{n+\frac{1}{2}} \underline{\hat{v}} \cdot\left(\underline{\underline{F}}_{k+1}^{n+\frac{1}{2}}\right)^{-1} \cdot \underline{\hat{v}} J_{k+1}^{n+\frac{1}{2}} d \hat{\Gamma}=0, \forall \underline{\hat{v}} \in \hat{\mathcal{V}}_{h},
\end{aligned}
$$

4. Given $\Omega_{k+1}^{n+1}=\hat{\Omega}+\underline{y}_{k+1}^{n+1}$ and $\left(\underline{u}^{s}\right)_{k+1}^{n+1}=\left(\underline{y}_{k+1}^{n+1}-\right.$ $\left.\underline{y}^{n}\right) / \Delta t$, solve for $p_{k+1}^{n+1}, \underline{w}_{k+1}^{n+1}$ such that

$$
\begin{gathered}
\int_{\Omega_{k+1}^{n+1}} q\left(\nabla \cdot \underline{w}_{k+1}^{n+1}+\frac{1}{(J M f)_{k+1}^{n+1} \Delta t}\left(p_{k+1}^{n+1}-p^{n}\right)\right) d \Omega \\
+\int_{\Omega_{k+1}^{n+1}} q\left(b-\frac{f_{k+1}^{n+1}}{M f_{k+1}^{n+1}{ }^{2}}\left(p_{k+1}^{n+1}-p_{0}\right)\right) \nabla \cdot\left(\underline{u}^{s}\right)_{k+1}^{n+1} d \Omega \\
=\int_{\Omega_{k+1}^{n+1}} q s^{n+1} d \Omega, \forall q \in \mathcal{Q}_{h} \\
\int_{\Omega_{k+1}^{n+1}} \underline{K}^{-1} \underline{w}_{k+1}^{n+1} \cdot \underline{v} d \Omega-\int_{\Omega_{k+1}^{n+1}} p_{k+1}^{n+1} \nabla \cdot \underline{v} d \Omega \\
=-\int_{\Gamma_{p}^{n+1}}^{n+1} p_{b c}^{n+1} \underline{\nu} \cdot \underline{v} d \Gamma, \forall \underline{v} \in \mathcal{V}_{h},
\end{gathered}
$$

5. Given $\underline{y}_{k+1}^{n+1}$ and $p_{k+1}^{n+1}$, compute $\tilde{m}_{k+1}^{n+1}$ from (14)

6. Relaxation step: $m_{k+1}^{n+1}=\omega_{k} \tilde{m}_{k+1}^{n+1}+\left(1-\omega_{k}\right) m_{k}^{n+1}$

Steps 3 to 6 are repeated for iterations $k=0 \ldots N_{i t}$ until convergence is reached at $N_{i t}$. The relaxation parameter $\omega_{k}$ of the last step is computed with the Aitken method [26]. Note that when the porosity is constrained to be positive by penalization, the fluid system becomes nonlinear in $p$. It is then solved iteratively as above with a quasi-linear approach. Note also that we neglect $m$ compared to $\rho_{0}$ in the inertia term.

\subsection{Numerical tests}

In this section we present two test problems to illustrate the behavior of this poroelastic model under large deformations: swelling and drainage of a cube. In addition to those given in the text, a list of all the parameters ${ }^{1}$ used

\footnotetext{
${ }^{1}$ See definition of viscosity parameter $\eta$ in Section 4
} 
in these simulations is reported in Table 1.

In the swelling test, no external force is applied on the skeleton but a fluid pressure gradient is imposed between two opposite faces (see Figure 1). For the skeleton, normal displacements are zero for the planes $x=0, y=0$ and $z=0$. On the other faces $P_{\mathrm{v}}=0$. For the fluid, there are neither sinks nor sources. The pressure $p_{b c}$ on the inlet face $x=0$ increases from zero to a limit value of $1 \mathrm{kPa}\left(p_{b c}=10^{3}\left(1-\exp \left(-t^{2} / 0.25\right)\right) \mathrm{Pa}\right)$. On the outlet face $(x=1$ in the initial configuration), pressure is zero. A null flux condition is applied on the four other faces. As a consequence, fluid enters in the medium from the inlet face. Velocity increases as the inlet pressure rises. The cube swells like a sponge undergoing large deformation as shown in Figure 1. When the inlet pressure has reached its limit value, a steady state takes place: the elastic forces are in equilibrium with the pressure increase due the added fluid. Although pressure decreases roughly linearly with $x$, the added fluid mass decreases nonlinearly (see the plots of $p$ and $m$ over time for three points across the cube in Figure 1). The perfusion velocity goes mainly in the $x$ direction, but unlike for a purely Darcy flow, it is not homogeneous in space (see the arrows on the deformed cube).

By contrast, in the drainage test, an external pressure of the order of magnitude of the left ventricle pressure in systole $P_{\mathrm{v}}=10 \mathrm{kPa}$ is gradually applied on all the faces of the cube $\left(P_{\mathrm{v}}=10^{4}\left(1-\exp \left(-t^{2} / 0.04\right)\right) \mathrm{Pa}\right)$. A null flux condition is applied on all the faces but the pores are connected to a pressure sink term $s=-\beta(p-$ $\left.p_{\text {sink }}\right)$. Here $\beta=10^{-4} \mathrm{~s}^{-1} \mathrm{~Pa}^{-1}$ and $p_{\text {sink }}=0 \mathrm{~Pa}$. As a result, the fluid is drained out of the sponge and the cube shrinks. If the external pressure is high enough to drain all the fluid, the penalization introduced in equation (30) prevents $\phi$ from being negative. The results thus show two different phases, as can be seen in Figure 2. In the first phase, fluid is drained until the porosity is nearly zero, at which point $m \approx-\phi_{0}$. In the second phase, the skeleton behaves as its solid component (we only see the compressibility effects through the bulk $K$ ). The theoretical steady state is thus given by the equilibrium of the volume terms in (13), i.e.

$K(1-1 / J)+M b \frac{\phi_{0}}{\rho_{0}^{f}}\left(f+(J-1) f^{\prime}\right)+\frac{1}{2} M\left(\frac{\phi_{0}}{\rho_{0}^{f}}\right)^{2} f^{\prime}=-P_{\mathrm{v}}$

For the value of $P_{\mathrm{v}}$ considered this gives $J=0.8604$, and we obtain this value with the same precision in the numerical results. Note that in this test the physical quantities are homogeneous in space.

\section{Application to cardiac perfusion}

Cardiac perfusion is the flow of the blood through the myocardium. In the next section, some basic physiological notions are briefly recalled. Then we explain how the
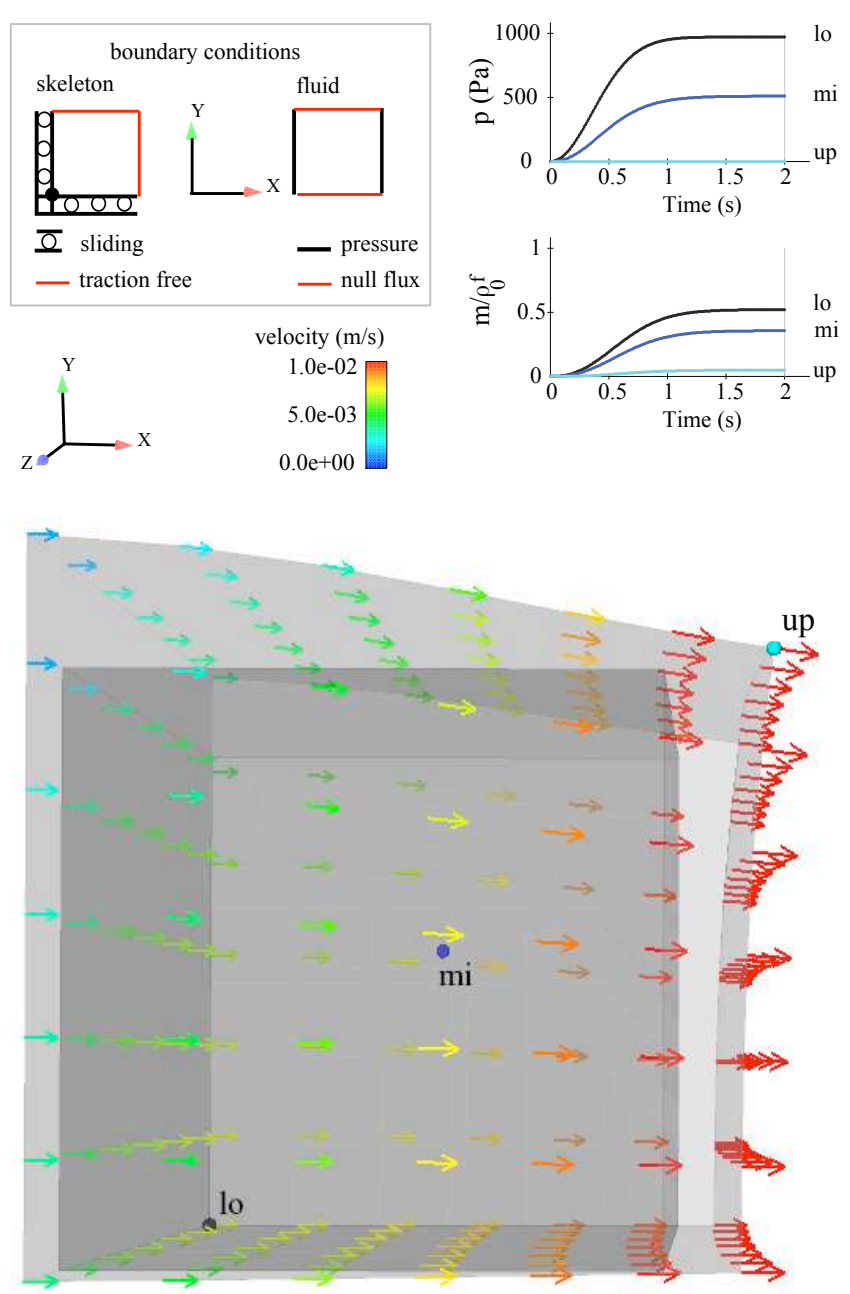

Fig. 1 Swelling test of a cube. No external force is applied on the skeleton but a fluid pressure gradient is imposed between two opposite faces whereas a null flux condition is applied on the four other faces. Dark grey represents the initial cube, and light grey the deformed cube. The arrows are the velocity vectors, colored by their magnitude. Pressure and mass are plotted against time for three points (the lower point attached to the inlet face in black, the middle point in blue and the upper point attached to the outlet face in cyan).

poroelastic model introduced above can be extended to describe the cardiac perfusion in a simplified manner.

\subsection{Physiological description}

Oxygen-rich blood coming from the lungs enters the left ventricle at low pressure. Subsequently the active contraction of the myocardium, depicted in paragraph 4.2 , forces the blood into the aorta which then branches into many smaller arteries which run throughout the body. Some of these arteries, named coronaries, supply the myocardium with blood, and thus ensure its oxygenation. Constriction and dilation of the coronary arteries, 


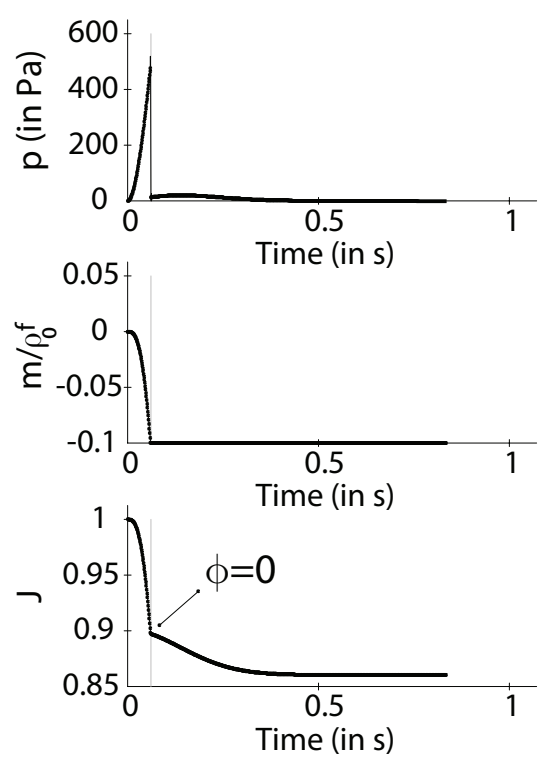

Fig. 2 Drainage test of the porous medium by applying an external pressure on the exterior of the skeleton. Fluid is first drained out of the media until $\phi=0$. Then the medium is compressed according to the compressibility of the solid phase.

Table 1 Parameter table for the swelling and drainage tests

\begin{tabular}{|c|c|c|c|}
\hline Parameter & Units & Swelling & Drainage \\
\hline$\kappa_{1}$ & $\mathrm{~Pa}$ & $2.10^{3}$ & $2.10^{3}$ \\
\hline$\kappa_{2}$ & $\mathrm{~Pa}$ & 33 & 33 \\
\hline$\eta$ & Pa.s & 68 & 68 \\
\hline$K$ & $\mathrm{~Pa}$ & $2.210^{5}$ & $2.210^{5}$ \\
\hline$M$ & $\mathrm{~Pa}$ & $2.1810^{5}$ & $2.1810^{5}$ \\
\hline$b$ & - & 1 & 1 \\
\hline$\kappa_{0}$ & $\mathrm{~Pa}$ & 0.01 & 0.01 \\
\hline$\rho_{0}^{s}$ & $\mathrm{~kg} \cdot \mathrm{m}^{-3}$ & $10^{3}$ & $10^{3}$ \\
\hline$\rho_{0}^{f}$ & $\mathrm{~kg} \cdot \mathrm{m}^{-3}$ & $10^{3}$ & $10^{3}$ \\
\hline$\phi_{0}$ & - & 0.1 & 0.1 \\
\hline$p_{0}$ & $\mathrm{~Pa}$ & 0 & 0 \\
\hline$\underline{K}$ & $\mathrm{~m}^{2} \mathrm{~Pa}^{-1} \mathrm{~s}^{-1}$ & $10^{-7} \underline{I}$ & $2.510^{-6} \underline{I}$ \\
\hline$\left|\overline{\overline{\Omega_{0}}}\right|$ & $\mathrm{m}^{3}$ & $10^{-\overline{\overline{6}}}$ & $10^{-\overline{\overline{9}}}$ \\
\hline$\Delta t$ & $\mathrm{~s}$ & $10^{-3}$ & $10^{-4}$ \\
\hline
\end{tabular}

governed primarily by local regulatory mechanisms, control the amount of blood through the myocardium. The blood carrying oxygen combined with hemoglobin goes from big coronaries to arterioles and finally to capillaries. The hemoglobin is then combined with carbon dioxide. Finally, venules and veins carry deoxygenated blood back to the right compartment of the heart. Unlike most of the organs, this flow of blood occurs in a very dynamic network of vessels, due to the beating of the heart.

\subsection{Active tissue modeling}

In our above discussions on poroelasticity we have considered a passive material. In order to represent the ac- tive behavior of the cardiac tissue with the contraction induced by electrical activation, we extend the approach described in [24] to incorporate the contribution of the porous flow.

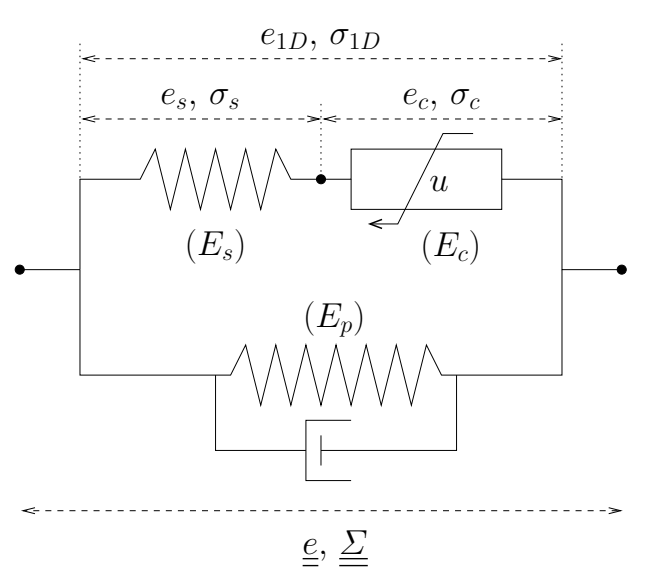

Fig. 3 Hill-Maxwell rheological model

More specifically, we use the rheological model of Hill-Maxwell type shown in Figure 3 where the passive element denoted by $E_{p}$ behaves according to the poroelastic constitutive law (13). We denote the corresponding poroelastic stress tensor by $\underline{\underline{\Sigma}}_{p}(\underline{\underline{e}}, m)$, and we henceforth reserve the plain notation $\underline{\Sigma}$ for the complete stress tensor. The dashpot symbol attached to $E_{p}$ signifies that a viscous stress $\eta \underline{\underline{\dot{e}}}$ is added to $\underline{\underline{\underline{\Sigma}}} p$, with $\eta$ a viscosity parameter corresponding to a simple Rayleigh damping. The active part of the behavior is represented by $E_{c}$ which obeys the active dynamic constitutive equation proposed in [27] - and further substantiated in [24,28, 29] - which incorporates an input variable $u$ representing the electrical activation - corresponding to a normalized concentration of calcium bound on the troponin- $\mathrm{C}$ - with $u>0$ during contraction and $u<0$ during active relaxation. The modeling of $u$ is further discussed in [30,31]. Finally, the series element $E_{s}$ is associated with a linear stress-strain law $\sigma_{s}=E_{s} e_{s}$.

The detailed interpretation of the rheological schematic of Figure 3 is as in [24]. Namely, in the active branch the stress-strain law is one-dimensional along the fiber directions represented by the unit tangent vector $\underline{n}$, and due to large strains we have the multiplicative composition rule

$$
1+2 e_{1 D}=\left(1+2 e_{c}\right)\left(1+2 e_{s}\right)
$$

for $e_{1 D}$ defined as

$$
e_{1 D}=\sum_{i, j} e_{i j} n_{i} n_{j}
$$

Moreover, the equilibrium equation in this branch is given by

$$
\sigma_{1 D}=\frac{\sigma_{c}}{1+2 e_{s}}=\frac{\sigma_{s}}{1+2 e_{c}} .
$$


Finally, the complete expression of the 3D stress tensor reads

$$
\underline{\underline{\Sigma}}=\underline{\underline{\Sigma}} p(\underline{\underline{e}}, m)+\eta \underline{\underline{\dot{e}}}+\sigma_{1 D} \underline{n} \otimes \underline{n},
$$

where " $\otimes$ " denotes tensorial product.

\subsection{Fluid modeling}

As recalled in Section 4.1, heart perfusion consists of a flow through coronary arteries, arterioles, capillaries, venules and veins. Several compartments could be modeled individually by a porous medium flow and coupled through exchange terms (see e.g. [20]). Our experience with this kind of approach is that it can be extremely difficult to parametrize each compartment in order to get meaningful behaviors in realistic 3D configurations, in particular to avoid non-physiological backflows. In the present work, we use a single compartment poroviscoelastic model, which is supposed to only describe small arteries and capillaries. The venous network is modeled as a simple sink term in the porous flow equations. In future works, the porous compartment will be coupled to three dimensional models of large coronaries. But for the time being, the porous flow is assumed for simplicity to be fed by a distributed source term. Thus in the second equation of (32), the quantity $s$ is defined by:

$$
s=\beta_{a}\left(p_{a}-p\right)-\beta_{v}\left(p-p_{v}\right),
$$

where $\beta_{a}, p_{a}$ and $\beta_{v}, p_{v}$ are given constants, respectively characterizing the small arteries and small veins compartments.

\subsection{Numerical results}

In this section, we present a simulation of the model described above on a three-dimensional analytical geometry that mimics the left ventricle. Fibers are embedded with an orientation that varies nonlinearly across the wall [24]. The activation of the muscle occurs during $0.25 \mathrm{~s}$, after which the muscle relaxes so that the heart beat lasts $0.8 \mathrm{~s}$. The contraction is such that the ejection fraction is normal (around 50\%). The top part that would be attached to the atrium is constrained to zero displacement. On the epicardium, the effect of the pericardium is neglected and thus $P_{\mathrm{v}}=0$. To investigate the effect of the contraction decoupled from the ventricular pressure, there is no valve in this model and the ventricle is unloaded $\left(P_{\mathrm{v}}=0\right.$ on the endocardium). On the fluid side, blood cannot enter into or leak from the ventricle (a null flux condition is applied on the whole boundary). Instead, blood enters in the myocardium from a distributed source with $p_{a}=2.7 \mathrm{kPa}$ which corresponds to a typical small artery pressure of $20 \mathrm{mmHg}$, and leaves through the venous side at a pressure of $10 \mathrm{mmHg}$ modeled as a distributed sink with $p_{v}=1.3 \mathrm{kPa}$. The pressure differential across the capillary bed is thus of $10 \mathrm{mmHg}$.
These values are consistent with literature data for the myocardium and skeletal muscles [32-34]. The inverse of the volume resistance to flow from the small arteries and into the small veins is $310^{-5} \mathrm{~Pa}^{-1} \mathrm{~s}^{-1}$, chosen so that the average flow rate for the whole myocardium corresponds to $4 \%$ of a normal cardiac output [35]. The blood porosity $\phi_{0}$ is $0.15[36,37]$, representing here the volume fraction of blood in the capillaries, which is ten times larger than the ones of the other small vessels [38]. The permeability $\underline{K}$ is isotropic and homogeneous with a scalar value of $2 \overline{\overline{10}} 0^{-9} \mathrm{~m}^{2} \mathrm{~Pa}^{-1} \mathrm{~s}^{-1}$ [39]. The simulation was run with a mesh of 15,000 elements and a time step of $5 \mathrm{~ms}$. Periodicity of the solution was achieved after 3-4 cycles. The number of fixed-point iterations necessary to achieve convergence of a relative residual on the mass $m$ of $10^{-4}$ was typically 4 , and up to 8 at the peak of the contraction.

The resulting time-averaged behavior is a system in which blood flows continuously from the small arteries into the capillaries, and from the capillaries into the small veins, at a flow rate of $4 \mathrm{~mL} \mathrm{~s}^{-1}$, a mean pressure of $15 \mathrm{mmHg}(2 \mathrm{kPa})$ and a muscle volume of $260 \mathrm{~mL}$. Figure 4 shows the variation in time around this state. During contraction, the volume of the myocardium decreases, while it increases during relaxation. This phenomenon has been reported in [40] and references herein. The contraction of the fibers induces a rise of the pressure. As a consequence, the flow from the small arteries into the capillaries $q_{a}$ is considerably reduced - which corresponds to the so called flow impediment ([3] and references herein) - and blood is squeezed out of the capillaries (lowering of $m$ ) into the small veins (rise of $q_{v}$ ). During relaxation, the opposite happens: $m$ rises as $q_{a}$ increases and $q_{v}$ decreases, filling up the capacitance of the capillaries. The mainly systolic flow in the small arteries and mainly diastolic flow in the small veins are characteristic of the coronary network, and consistent with the measured velocities in small arteries and small veins in the left ventricle given in [38].

Furthermore, as can be seen in Figure 5, pressure and mass vary nonlinearly across the myocardium wall. During contraction, pressure, and consequently flows as well, is more affected by the contraction in the subendocardium than in the subepicardium. The model is thus able to reproduce the fact that flow impediment is known to be higher in the subendocardium than in the subepicardium [41,38]. In addition, Figure 6 shows that the solution is heterogeneous in the different regions of the myocardium, which supports the choice of such a threedimensional model. During the cardiac cycle, the myocardium undergoes large deformations. The displacement of the apex between the relaxed and the contracted states is here of $2.4 \mathrm{~cm}$, which corresponds to $24 \%$ of the initial length. 


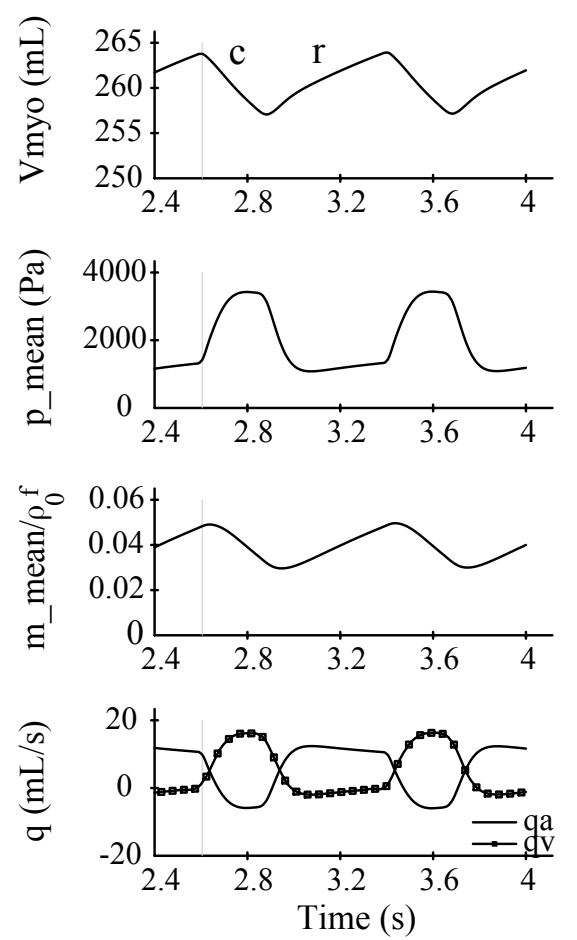

Fig. 4 Variations over two cardiac cycles of the volume of the myocardium with the contraction (c) and relaxation (r) phases, the pressure averaged over the volume, the added blood mass averaged over the volume, and the flow rates that come from the small arteries $\left(q_{a}\right)$ and leave into the small veins $\left(q_{v}\right)$.

This simulation shows that key phenomena that characterize the cross-talk between myocardium and coronary flow are described by this model. However the former presents several limitations. The capacitance of the arteries and veins is neglected, as a consequence flow in the small arteries is negative during part of the contraction. Nevertheless, negative flows have been recorded in coronary arteries $[38,42]$. Furthermore, the pressures in the small arteries and veins are taken constant in time and homogeneous. The pulsatility in the pressure at these levels is usually considered to be negligible [43] but these pressures are likely to be inhomogeneous. To improve the model, one could couple it with a distributed network of the larger arteries and veins. Homogeneous and constant inverse resistance parameters as well as a homogeneous reference porosity are chosen for simplicity. This is further motivated by the fact that their variations are a matter of debate [3].

\section{Conclusion}

We have proposed a poroelastic model accounting for large strains and compatible with incompressible conditions. The modeling, numerical implementation and
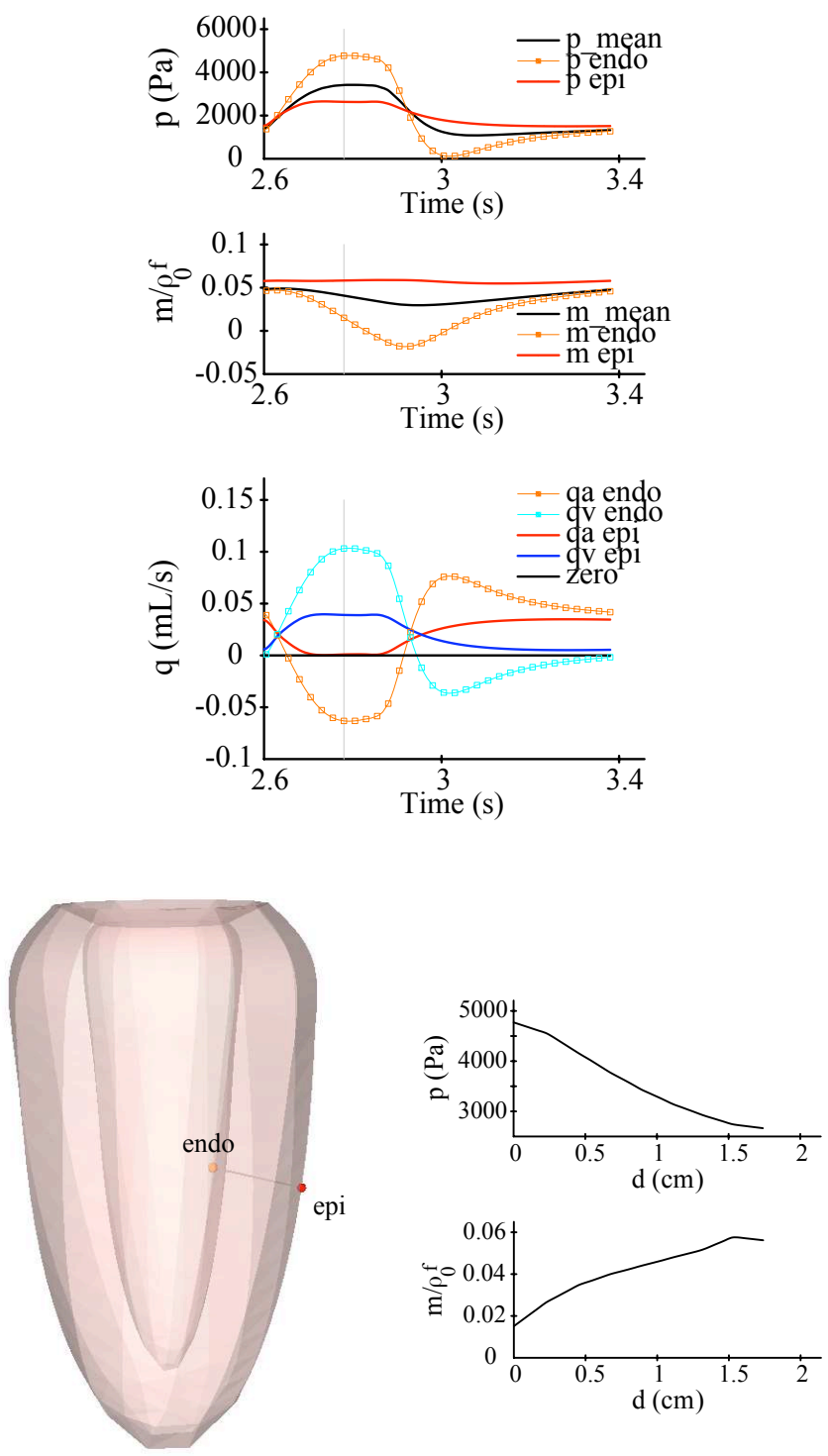

Fig. 5 Mean pressure and mass are traced over one cardiac cycle (upper left graphs). Pressure, mass, and arterial and venous flow rates are traced for two different locations - endocardium (endo) and epicardium (epi). Their exact location in the myocardium is shown in the 3D figure. The pressure and mass gradients across the myocardium wall between these two points are represented on the lower right graphs ( $\mathrm{d}$ is the distance from the endocardium point).

some verifications were presented in this article. A special emphasis was placed on the compatibility with thermodynamics principles. The model has displayed an excellent behavior when used in the verification tests characteristic of essential poroleastic phenomena with large displacements and strains, including when undergoing complete drainage of the fluid.

As far as cardiac perfusion is concerned, the major difficulty lies in the complexity of the physiological phenomena and the lack of sufficiently detailed mea- 


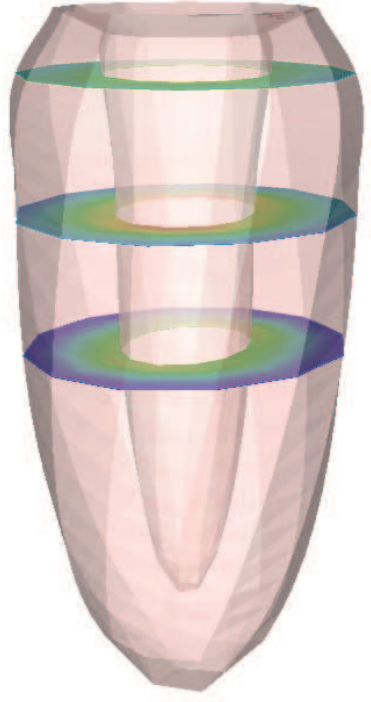

$\mathrm{p}(\mathrm{Pa})$

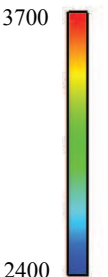

Fig. 6 Myocardium with pressure contours shown on three slices.

surements to validate the various modeling assumptions. Nevertheless, in spite of the relative simplicity of the model, the proposed numerical experiments have shown that important mechanisms of perfusion appear to be adequately captured. We therefore believe that this model can be viewed as a tool to investigate the mechanisms of cross-talk between the myocardium and coronary flow, taking into account both the stresses generated by the contraction as in this study, as well as the effect of the left ventricular pressure, which will be the object of future work.

The present study can be extended in many directions. For example: coupling of the porous flow with models representing the circulation in larger arteries; simulation of some characteristic pathologies, e.g. an infarcted area as reflected in a flow impediment combined with a decrease of local muscular contractility; modeling of the supply of the nutrients necessary for the cardiac muscle activity; validation and calibration with clinical data.

Acknowledgments: the authors are very thankful to Philippe Moireau (INRIA) for several discussions on this subject, and for his help in implementing the poroelasticity model.

\section{References}

1. J. Spaan, C. Kolyva, J. van den Wijngaard, R. ter Wee, P. van Horssen, J. Piek, M. Siebes, Coronary structure and perfusion in health and disease, Philosophical Transactions of the Royal Society A 366 (1878) (2008) 31373153.

2. P. Horssen, J. P. H. M. Wijngaard, M. Siebes, J. A. E. Spaan, Improved regional myocardial perfusion measurement by means of an imaging cryomicrotome, in: 4th
European Conference of the International Federation for Medical and Biological Engineering, Springer, 2009, pp. 771-774.

3. N. Westerhof, C. Boer, R. R. Lamberts, P. Sipkema, Cross-Talk Between Cardiac Muscle and Coronary Vasculature, Physiol. Rev. 86 (4) (2006) 1263-1308.

4. N. Smith, G. Kassab, Analysis of coronary blood flow interaction with myocardial mechanics based on anatomical models, Phil. Trans. R. Soc. Lond. A 359 (2001) 1251-1262.

5. N. Smith, A computational study of the interaction between coronary blood flow and myocardial mechanics, Physiological Measurement 25 (4) (2004) 863-877.

6. K. Terzaghi, Theoretical Soil Mechanics, John Wiley and Sons, New-York, 1943.

7. M. A. Biot, Theory of propagation of elastic waves in a fluid-saturated porous solid. II higher frequency range, J. Acoust. Soc. Am. 28 (1956) 179-191.

8. M. A. Biot, Theory of finite deformations of porous solids, Indiana Univ. Math. J. 21 (1972) 597-620.

9. K. May-Newman, A. D. McCulloch, Homogenization modeling for the mechanics of perfused myocardium, Progress in Biophysics \& Molecular Biology 69 (1998) 463-481.

10. E. Almeida, R. Spilker, Finite element formulations for hyperelastic transversely isotropic biphasic soft tissues, Computer Methods in Applied Mechanics and Engineering 151 (3-4) (1998) 513-538.

11. Z. Yang, P. Smolinski, Dynamic finite element modeling of poroviscoelastic soft tissue, Computer Methods in Biomechanics and Biomedical Engineering 9 (1) (2006) $7-16$.

12. R. Borja, On the mechanical energy and effective stress in saturated and unsaturated porous continua, International Journal of Solids and Structures 43 (6) (2006) 1764-1786.

13. S. Badia, A. Quaini, A. Quarteroni, Coupling Biot and Navier-Stokes equations for modelling fluid-poroelastic media interaction, to appear in Journal of Computational Physics.

14. N. Koshiba, J. Ando, X. Chen, T. Hisada, Multiphysics simulation of blood flow and LDL transport in a porohyperelastic arterial wall model, Journal of biomechanical engineering 129 (2007) 374.

15. V. Calo, N. Brasher, Y. Bazilevs, T. Hughes, Multiphysics model for blood flow and drug transport with application to patient-specific coronary artery flow., Comput. Mech. 43 (1) (2008) 161-177.

16. P. Feenstra, C. Taylor, Drug transport in artery walls: A sequential porohyperelastic-transport approach, Computer Methods in Biomechanics and Biomedical Engineering 12 (3) (2009) 263-276.

17. J. M. Huyghe, D. H. van Campen, Finite deformation theory of hierarchically arranged porous solids: I. Balance of mass and momentum, Int. J. Engng Sci. 33 (13) (1991) 1861-1871.

18. J. M. Huyghe, D. H. van Campen, Finite deformation theory of hierarchically arranged porous solids: II. Constitutive behaviour, Int. J. Engng Sci. 33 (13) (1991) 1861-1871.

19. R. Cimrman, E. Rohan, Modelling heart tissue using a composite muscle model with blood perfusion, in: Bathe, 
KJ (Ed.), Computational Fluid and Solid Mechanics, 2nd MIT Conference, 2003, pp. 1642-1646.

20. W. Vankan, J. Huyghe, J. Janssen, A. Huson, A finite element mixture model for hierarchical porous media., Int. J. Numer. Meth. Engng. 40 (1997) 193-210.

21. O. Coussy, Mechanics of porous continua, Wiley, NewYork, 1995.

22. P. de Buhan, X. Chateau, L. Dormieux, The constitutive equations of finite-strain poroelasticity in the light of a micro-macro approach, Eur. J. Mech. A/Solids 17 (6) (1998) 909-922.

23. P. G. Ciarlet, G. Geymonat, Sur les lois de comportement en élasticité non linéaire, C.R.A.S, Série II 295 (1982) 423-426.

24. J. Sainte-Marie, D. Chapelle, R. Cimrman, M. Sorine, Modeling and estimation of the cardiac electromechanical activity, Computers \& Structures 84 (2006) 17431759.

25. F. Brezzi, M. Fortin, Mixed and hybrid finite element method, Springer, New-York, 1991.

26. B. Irons, R. Tuck, A version of the Aitken accelerator for computer implementation, Int. J. Num. Meth. Engng. 1 (1969) 275-277.

27. J. Bestel, F. Clément, M. Sorine, A biomechanical model of muscle contraction, in: Lectures Notes in Computer Science, Vol. 2208, Eds W.J. Niessen and M.A. Viergever, Springer-Verlag, 2001, pp. 1159-1161.

28. P. Krejčí, J. Sainte-Marie, M. Sorine, J. Urquiza, Solutions to muscle fiber equations and their long time behaviour, Nonlinear Analysis: Real World Analysis 7 (4) (2005) 535-558.

29. D. Chapelle, P. Le Tallec, P. Moireau, Mechanical modeling of the heart contraction, in preparation.

30. D. Chapelle, M. Fernández, J.-F. Gerbeau, P. Moireau, J. Sainte-Marie, N. Zemzemi, Numerical simulation of the electromechanical activity of the heart, in: FIMH, Vol. 5528 of Lecture Notes in Computer Science, 2009, pp. 357-365.

31. M. Boulakia, S. Cazeau, M. A. Fernández, J.-F. Gerbeau, N. Zemzemi, Mathematical Modeling of Electrocardiograms: A Numerical Study, Research Report RR-6977, INRIA (2009). URL http://hal.inria.fr/inria-00400490/en/

32. D. Zinemanas, R. Beyar, S. Sideman, An integrated model of LV muscle mechanics, coronary flow, and fluid and mass transport, Am J Physiol Heart Circ Physiol 268 (2) (1995) H633-645.

33. G. S. Kassab, K. N. Le, Y.-C. B. Fung, A hemodynamic analysis of coronary capillary blood flow based on anatomic and distensibility data, Am J Physiol Heart Circ Physiol 277 (6) (1999) H2158-2166.

34. K. Fronek, B. Zweifach, Microvascular pressure distribution in skeletal muscle and the effect of vasodilation, Am J Physiol 228 (3) (1975) 791-796.

35. R. Berne, M. Levy, Cardiovascular Physiology, St Louis, Mosby, 2001.

36. F. Gonzalez, J. B. Bassingthwaighte, Heterogeneities in regional volumes of distribution and flows in rabbit heart, Am J Physiol Heart Circ Physiol 258 (4) (1990) H10121024.

37. K. May-Newman, C. Chen, R. Oka, R. Haslim, A. DeMaria, Evaluation of myocardial perfusion using threedimensional myocardial contrast echocardiography, in:
Nuclear Science Symposium Conference Record, 2001 IEEE, Vol. 3, 2001, pp. 1691-1694 vol.3.

38. D. Ghista, E. Ng, Cardiac Perfusion and Pumping Engineering, World Scientific, 2007.

39. J. M. Huyghe, T. Arts, D. H. van Campen, R. S. Reneman, Porous medium finite element model of the beating left ventricle, Am J Physiol Heart Circ Physiol 262 (4) (1992) H1256-1267.

40. H. Ashikaga, B. A. Coppola, K. G. Yamazaki, F. J. Villarreal, J. H. Omens, J. W. Covell, Changes in regional myocardial volume during the cardiac cycle: implications for transmural blood flow and cardiac structure, Am J Physiol Heart Circ Physiol 295 (2) (2008) H610-618.

41. M. Goto, A. E. Flynn, J. W. Doucette, C. M. Jansen, M. M. Stork, D. L. Coggins, D. D. Muehrcke, W. K. Husseini, J. I. Hoffman, Cardiac contraction affects deep myocardial vessels predominantly, Am J Physiol Heart Circ Physiol 261 (5) (1991) H1417-1429.

42. D. Gregg, H. Green, Registration and interpretation of normal phasic inflow into a left coronary artery by an improved differential manometric method, Am J Physiol 130 (1940) 114-125.

43. W. Nichols, M. O'Rourke, McDonald's blood flow in arteries, Hodder Arnold, 2005. 